\title{
The Belle II Experiment at the SuperKEKB
}

\author{
Chang-Zheng Yuan (for the Belle II Collaboration ) $)^{1,2, \star ~}$ \\ ${ }^{1}$ Institute of High Energy Physics, Chinese Academy of Sciences, Beijing 100049, China \\ ${ }^{2}$ University of Chinese Academy of Sciences, Beijing 100049, China
}

\begin{abstract}
Belle II experiment at the SuperKEKB collider is a major upgrade of the Belle experiment at the KEKB asymmetric $e^{+} e^{-}$collider at the KEK. The experiment will focus on the search for new physics beyond the standard model via high precision measurement of heavy flavor decays and search for rare signals. In this talk, we present the status of the SuperKEKB collider and the Belle II detector.
\end{abstract}

\section{Introduction}

The two B factories, Belle at the KEKB collider at KEK [1] and BaBar at the PEP II collider at SLAC [2], have been operating very successfully for more than ten years [3]. Altogether, about $1.5 \mathrm{ab}^{-1}$ data in the vicinity of the $\Upsilon(4 S)$ resonance were accumulated, and these data were used in extensive studies of the B-physics, charm physics, tau physics, as well as hadron spectroscopy and various searches for possible hints of new physics, such as lepton number violation, low mass Higgs-like states, and dark matters.

While most results from B factories are in good consistency with the expectations from the Standard Model (SM), there are a few measurements that show discrepancies at around three standard deviation level from the SM predictions. One of the interesting examples is the possible observation of the violation of the lepton universality in semileptonic B decays, if confirmed, very probably, there is contribution from non-SM physics [4].

Much larger data sets are needed to investigate whether these are hints for New Physics (NP) or merely statistical fluctuations. The main purpose of a super flavor factory, SuperKEKB, is to accumulate a much larger data set than at the B factories, to pin down the experimental uncertainties, and to confirm or deny the discrepancies from the SM predictions $[4,5]$.

The KEKB collider has achieved world record luminosity of $2.1 \times 10^{34} \mathrm{~cm}^{-2} \mathrm{~s}^{-1}$ and the Belle experiment accumulated slightly more than $1 \mathrm{ab}^{-1}$ in about 10 years. To reach the physics goals of the next generation flavor-factory experiment, both the accelerator and the detector need to be upgraded significantly. The designed luminosity of the SuperKEKB is $8 \times 10^{35} \mathrm{~cm}^{-2} s^{-1}$ which is about 40 times higher than the KEKB, and the expected integrated luminosity at Belle II experiment is $50 \mathrm{ab}^{-1}$ in about 5 years running [6].

\footnotetext{
^e-mail: yuancz@ihep.ac.cn
} 


\section{SuperKEKB Collider}

The luminosity at an $e^{+} e^{-}$collider, $\mathcal{L}$, is given by

$$
\mathcal{L}=\frac{\gamma_{e^{ \pm}}}{2 e r_{e}}\left(1+\frac{\sigma_{y}^{*}}{\sigma_{x}^{*}}\right) \frac{I_{e^{ \pm}} \xi_{y}^{e^{ \pm}}}{\beta_{y}^{* e^{ \pm}}}\left(\frac{R_{L}}{R_{\xi_{y}}}\right)
$$

where $\gamma$ is the Lorentz factor, $\sigma_{y}^{*} / \sigma_{x}^{*}$ the beam size aspect ratio, $I$ the beam current, $\beta_{y}^{*}$ the vertical beta function at the interaction point, $\xi_{y}$ the beam-beam parameter, and $R_{L} / R_{\xi_{y}}$ a geometrical factor. The $e^{ \pm}$refers to the product of the corresponding quantities for the low energy positron (LER) and high energy electron (HER) beams.

In order to achieve a factor of 40 increase in the luminosity than at KEKB, all the above parameters have to be improved. The main increase in luminosity comes from a significantly smaller beam size at the interaction point (the so-called nano-beam scheme). The beta functions at the interaction point are reduced in $y$ direction from $5.9 / 5.9 \mathrm{~mm}$ to $0.27 / 0.31 \mathrm{~mm}$ for LER/HER, and in $x$ direction from $1200 / 1200 \mathrm{~mm}$ to $32 / 25 \mathrm{~mm}$.

The beam-beam parameter is proportional to $\sqrt{\beta^{*} / \epsilon}$, the emittance $\epsilon$ is reduced to keep the beambeam parameter at the same level as at KEKB. A reduction of the emittance from 18/24 nm to 3.2/(4.34.6) $\mathrm{nm}$ for LER/HER is obtained by installing a new electron source and a new damping ring, in addition to a redesign of the HER arcs. The last contribution to the luminosity gain comes from higher beam currents. They are increased from 1.64/1.19 A to 3.60/2.60 A for LER/HER.

To improve the lifetime of the LER beam, the beam energies are changed from $3.5 / 8 \mathrm{GeV}$ to $4 / 7 \mathrm{GeV}$, and the crossing angle of the two beams is increased from $22 \mathrm{mrad}$ to $83 \mathrm{mrad}$.

\section{Belle II Detector}

The increased background level (10-20 times more than at Belle) requires the Belle II detector to be able to handle the higher occupancy and radiation damage than at Belle. The about a factor of 10 increased event rate requires improved trigger, data acquisition, and computing systems. To reach higher precision measurement of some physics quantities requires improved low momentum $\mu$ identification and good neutrino reconstruction via missing energy. The components of the Belle detector are either significantly upgraded or replaced with new ones [6]. Figure 1 shows the structure of the Belle II detector.

The most challenging experimental requirement is the detection of the decay point of the shortliving B-mesons, relying on a high-performance vertex detector. The two inner layers of the Belle II vertex detector will use two layers of silicon pixel sensors (PXD) based on the DEPFET technology surrounded by four layers of double sided silicon strip detectors (SVD). With the excellent spatial resolution of the PXD, an impact parameter resolution in beam direction of $\sim 10 \mu \mathrm{m}$ can be achieved. The larger outer radius of the SVD compared to Belle gives an increase in efficiency of about $30 \%$ for the reconstruction of $K_{S} \rightarrow \pi^{+} \pi^{-}$decays inside the SVD. A precise measurement of the momentum and the energy loss $(\mathrm{dE} / \mathrm{dx})$ of charged tracks is provided by the central drift chamber (CDC) with a higher granularity, a new gas $-\mathrm{He} / \mathrm{C}_{2} \mathrm{H}_{6}$ and an improved readout system. It will start immediately after the vertex detector and cover range $16 \mathrm{~cm}<r<112 \mathrm{~cm}$ of distances from the beam axis.

Identification of hadrons is another key element for Belle II: it has to separate kaons from pions with high efficiency and very low fake rate. This will be provided by replacing the time-of-flight detector at Belle with the Time-of-Propagation (TOP) counter in the barrel region and the proximity focusing Cherenkov ring imaging counter with aerogel radiators in the endcaps. The TOP counter will measure the time that the internally reflected light travels down the quartz bar, together with two 


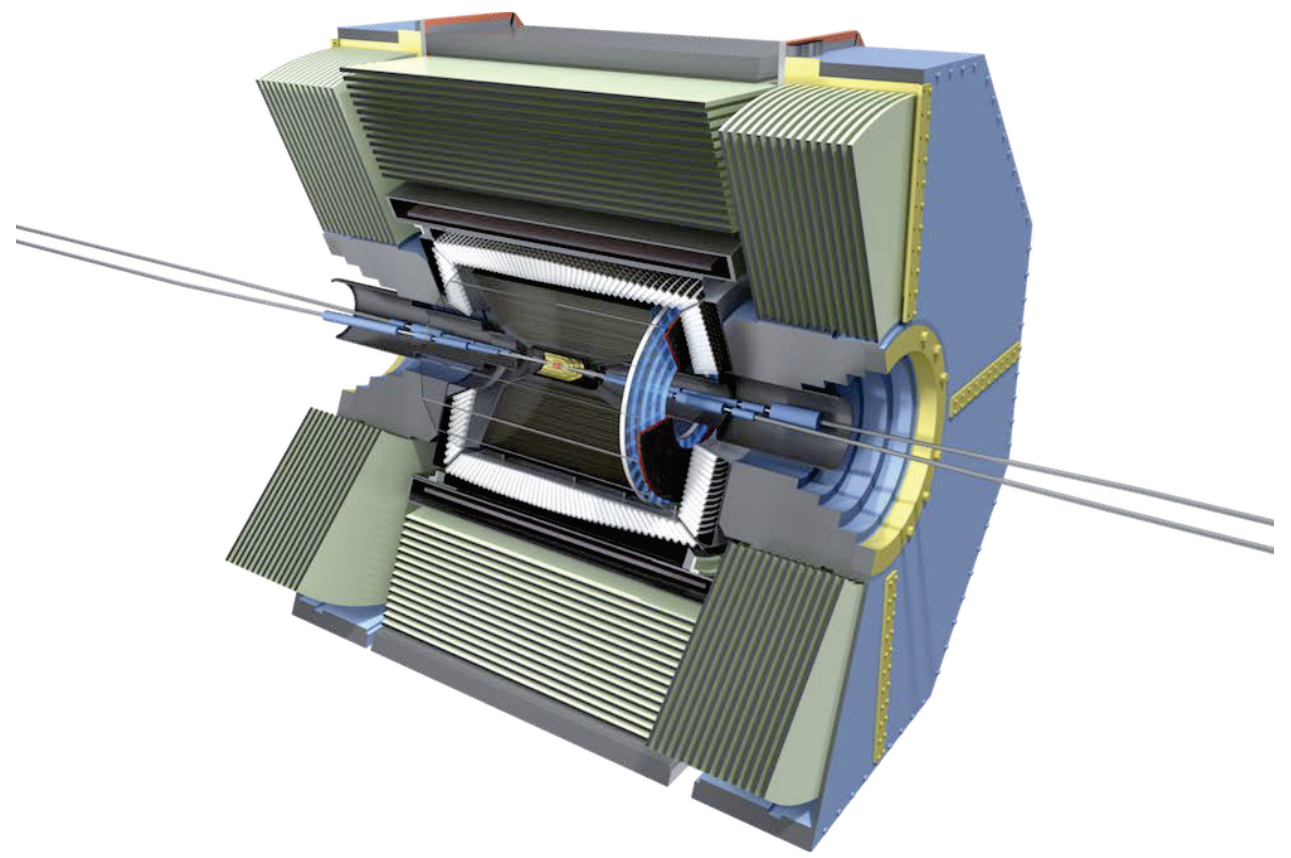

Figure 1. The Belle II detector.

spatial coordinates of the photon impact point at the bar end surface. A kaon identification efficiency of $>97 \%$ at a pion misidentification rate of $<1 \%$ is expected for almost the full momentum and angular ranges.

The electromagnetic calorimeter (ECL) will face a severe background increase. While the crystals of the Belle electromagnetic calorimeter will be reused, the readout electronics will be replaced by new ones with pipeline and waveform sampling.

Muons and $K_{L}$ mesons are identified by resistive plate chambers in the outer part of the Belle detector (KLM). For Belle II the endcap regions as well as the first three layers in the barrel region will be upgraded with scintillator strips to cope with the high background rates.

Computing system of the Belle II will use the newly developed BASF2 framework. It will have to handle an amount of data eventually corresponding to 50 times the Belle level in about 5 years' running. This means an amount of raw data of the order of $10^{10}$ events per year. Therefore, a distributed computing model based on the grid will be adopted.

\section{SuperKEKB Commissioning}

Beam commissioning is planned in three phases.

After more than five years of construction, the Phase 1 commissioning was carried out for 5 months from February to June 2016. The main goal of phase 1 commissioning is the machine tuning and vacuum scrubbing without the final focus superconducting magnet system (QCS) and the Belle II detector. At the interaction point, the so called "BEAST" detector was implemented instead of the Belle II detector to monitor the background level. The accelerator current reached $845 \mathrm{~mA}$ in HER, while $780 \mathrm{~mA}$ in LER on June 7 which are about half of the design currents. 
The Belle II detector was "rolled-in" to the collision point of the SuperKEKB accelerator in the afternoon of April 11th, 2017. The phase 2 commissioning will start in November 2017, and the first collision is expected in February 2018. In Phase 2, beam collision tuning starts with QCS and Belle II except the vertex detector. The target luminosity in Phase 2 is set to $1 \times 10^{34} \mathrm{~cm}^{-2} \mathrm{~s}^{-1}$.

The full Belle II detector will be ready in 2018, and the Phase 3 commissioning with the full detector will start late 2018, physics data are expected from phase 3 commissioning.

\section{Summary}

The upgrade of the B-factory at KEK is in progress. The accelerator, SuperKEKB, with a designed luminosity of $8 \times 10^{35} \mathrm{~cm}^{-2} \mathrm{~s}^{-1}$, will starts physics running by the end of 2018 , and will supply an integrated luminosity of $10 \mathrm{ab}^{-1}$ in 2021 , and $50 \mathrm{ab}^{-1}$ in 2024 . The Belle detector components are either upgraded or replaced by new ones to cope with the more challenging beam conditions and to improve the detector performance. For more details on the physics and the experiment, one may refer to Refs. [5] and [6].

\section{Acknowledgements}

The work is supported in part by National Natural Science Foundation of China (NSFC) under Contracts Nos. 11475187 and 11521505.

\section{References}

[1] A. Abashian et al. [Belle Collaboration], Nucl. Instrum. Meth. A 479, 117 (2002).

[2] B. Aubert et al. [BABAR Collaboration], Nucl. Instrum. Meth. A 479, 1 (2002).

[3] A. J. Bevan et al. [BaBar and Belle Collaborations], Eur. Phys. J. C 74, 3026 (2014).

[4] G. Ciezarek, M. Franco Sevilla, B. Hamilton, R. Kowalewski, T. Kuhr, V. Luth and Y. Sato, Nature 546, 227 (2017).

[5] T. Aushev et al., "Physics at Super B Factory," arXiv:1002.5012 [hep-ex]; and the B2TIP report to be published soon, see https://confluence.desy.de/display/BI/B2TiP+WebHome.

[6] T. Abe et al. [Belle II Collaboration], "Belle II Technical Design Report," arXiv:1011.0352 [physics.ins-det]. 